\title{
Content Analysis and Characterization of Medical Tweets During the Early Covid-19 Pandemic
}

Ross Prager ${ }^{1}$, Michael T. Pratte ${ }^{1}$, Rudy R. Unni ${ }^{1}$, Sudarshan Bala ${ }^{2}$, Nicholas Ng Fat Hing ${ }^{1}$, Kay Wu ${ }^{2}$, Trevor A. McGrath ${ }^{3}$, Adam Thomas ${ }^{4}$, Brent Thoma ${ }^{5}$, Kwadwo Kyeremanteng 6

1. Department of Medicine, University of Ottawa, Ottawa, CAN 2. Department of Medicine, McMaster University, Hamilton, CAN 3. Department of Radiology, University of Ottawa, Ottawa, CAN 4. Division of Critical Care, University of British Columbia, Vancouver, CAN 5. Emergency Medicine, University of Saskatchewan, Saskatoon, CAN 6. Division of Critical Care, University of Ottawa, Ottawa, CAN

Corresponding author: Ross Prager, rprag011@uottawa.ca

\section{Abstract}

\section{Objective}

The novel coronavirus disease 2019 (Covid-19) has infected millions worldwide and impacted the lives of many folds more. Many clinicians share new Covid-19-related resources, research, and ideas within the online Free Open Access to Medical Education (FOAM) community of practice. This study provides a detailed content and contributor analysis of Covid-19-related tweets among the FOAM community during the first months of the pandemic.

\section{Design, Setting, and Participants}

In this social media content analysis study, Twitter was searched from November 1, 2019, to March 21, 2020, for English tweets discussing Covid-19 in the FOAM community. Tweets were classified into one of 13 prespecified content categories: original research, editorials, FOAM resource, public health, podcast or video, learned experience, refuting false information, policy discussion, emotional impact, blatantly false information, other Covid-19, and non-Covid-19. Further analysis of linked original research and FOAM resources was performed. One-thousand (1000) randomly selected contributor profiles and those deemed to have contributed false information were analyzed.

\section{Results}

The search yielded 8541 original tweets from 4104 contributors. The number of tweets in each content category were: 1557 other Covid-19 (18.2\%), 1190 emotional impact (13.9\%), 1122 FOAM resources (13.1\%), 1111 policy discussion (13.0\%), 928 advice (10.9\%), 873 learned experience (10.2\%), 424 non-Covid-19 (5.0\%), 410 podcast or video (4.8\%), 304 editorials (3.6\%), 275 original research (3.2\%), 245 public health (2.9\%), 83 refuting false information (1.0\%), and 19 blatantly false $(0.2 \%)$.

Review began 02/01/2021 Review ended 02/19/2021 Published 02/27/2021

\section{() Copyright 2021}

Prager et al. This is an open access article distributed under the terms of the Creative Commons Attribution License CC-BY 4.0., which permits unrestricted use, distribution, and reproduction in any medium, provided the original author and source are credited.

\section{Conclusions}

Early in the Covid-19 pandemic, the FOAM community used Twitter to share Covid-19 learned experiences, online resources, crowd-sourced advice, and research and to discuss the emotional impact of Covid-19. Twitter also provided a forum for post-publication peer review of new research. Sharing blatantly false information within this community was infrequent. This study highlights several potential benefits from engaging with the FOAM community on Twitter.

Categories: Emergency Medicine, Medical Education, Infectious Disease

Keywords: covid-19, social media, pandemic, free open access medical education, medical education, knowledge translation

\section{Introduction}

Millions of cases of coronavirus disease 2019 (Covid-19) have been reported globally since the first known case in December 2019 [1-2]. Covid-19's worldwide impact has been recognized through its classification as a global pandemic by the World Health Organization (WHO) [3]. Covid-19's rapid spread has spurred healthcare workers, researchers, and members of the public to search for accurate and up-to-date information online. The rate of new Covid-19-related research, however, has challenged conventional methods of scientific knowledge dissemination (e.g. peer-reviewed journals), which do not always publish on rapid timelines [4]. In response, clinicians worldwide have turned to social media to debate new research while sharing their experiences and resources [5].

Social media use among clinicians is not a new phenomenon. In the past decade, an online community of practice has developed with the goal of sharing ideas, research, and learned experiences through freely 
published online resources [6-7]. Termed "Free Open Access to Medical Education" (FOAM), this movement has become a valuable resource for healthcare professionals and medical learners [7-8]. In addition to relaying explicit medical knowledge, it may also be an effective medium for transmitting tacit knowledge (experiential or process-based knowledge) [9]. Compared to traditional peer-reviewed journals, FOAM has variable publication and editorial processes relying heavily on post-publication peer review [10].

Optimizing knowledge translation is important during a pandemic, as critical decisions need to be made with limited evidence, and potentially practice-changing research can be published at any time. Within the FOAM community, Twitter is the most widely used social media platform to share ideas and discuss new research on Covid-19 [5]. On Twitter, contributors generate 'tweets' of up to 280 characters in length that can be tagged with searchable hashtags (\#) and can include images, website links, and documents. While the important role of Twitter during Covid-19 has been recognized by the scientific community [5], a detailed characterization of its use, strengths, and limitations, including accuracy of content, is needed. This is particularly important for a publicly accessed platform like Twitter that may be susceptible to misleading or false information.

The objective of this study was to characterize Covid-19-related Twitter use by the FOAM community and to describe its content, trends, and contributors. In addition, the potential role of Twitter in spreading misinformation was assessed. This research represents an important first step in evaluating Twitter as a platform for knowledge translation during rapidly evolving healthcare crises.

\section{Materials And Methods}

Research ethics board approval for research involving publicly available data is not required at our institutions. Our protocol was registered on the Open Science Framework (OSF) prior to the initiation of data collection (https://osf.io/3tx96/). The original data are also published on OSF. The study has been reported in keeping with the Strengthening the Reporting of Observational Studies in Epidemiology (STROBE) statement [11]. Patients or the public were not involved in the design, conduct, reporting, or dissemination plans of our research.

\section{Search strategy}

We searched Twitter on March 21, 2020, for tweets with relevant hashtags from November 1, 2019 (the month preceding the first reported Covid-19 case in Wuhan, China) to March 21, 2020, using a commercially available hashtag collating tool, Tweet Binder (Pamplona, Spain). The period between November 2019 to March 2020 was chosen, as it represented a time period when little consolidated information on Covid-19 was available to healthcare professionals despite concern surrounding Covid-19 being high. Hashtags were selected by consensus of the authors, several of whom were clinician members of the FOAM community. Tweets were included if they contained both a hashtag commonly used by healthcare professionals to discuss FOAM topics (\#FOAMed or \#meded or \#POCUS or \#FOAMcc or \#medtwitter) and a hashtag used to discuss Covid-19 (\#Covid19 or \#coronavirus or \#Covid or \#Covid-19). Alternatively, two Covid-19 FOAMspecific hashtags were also included (\#Covid4MDs or \#CovidFOAM). The search strategy was not casesensitive.

\section{Tweet analysis}

We extracted the following data: total number of original tweets (original text or image content), retweets (a reposted tweet without modification), reach (number of unique people who saw the tweet), impressions (number of times a tweet was liked or retweeted), the total number of contributors (accounts creating tweets), and median original tweets per original contributor.

The content of all original, English-language tweets was analyzed independently by one of five authors (MP, $\mathrm{SB}, \mathrm{KW}, \mathrm{NN}, \mathrm{RP}$ ). To assess inter-rater reliability, a duplicate extraction of 100 tweets was performed by all extractors. We assigned each tweet to one of 13 pre-determined 'content categories' created after consensus discussion between authors: 1) peer-reviewed original research study related to Covid-19, excluding editorials, commentaries, or perspective articles; 2) editorial, commentary, or perspective article published in a journal or repository relevant to Covid-19, including scientific article pre-prints; 3) FOAM resources pertaining to the care of Covid-19 patients; 4 ) public health agency website or university website (e.g. Centers for Disease Control and Prevention); 5) medical podcast or video relevant to Covid-19; 6) personal or learned experience caring for Covid-19 patients; 7) a statement or discussion refuting blatantly false or misleading information regarding Covid-19; 8) a discussion about policy or public health measures related to Covid-19; 9) a discussion of the personal or emotional impact of Covid-19; 10) a tweet that provided blatantly false or misleading information (defined through consensus agreement by two senior authors (RP and RU) that the tweet contained false or misleading information based on current medical consensus); 11) a tweet asking for advice or for others to share experience caring for Covid-19 patients; 12) other Covid-19 related tweets that did not fit in the other categories; 13) non-Covid-19-related tweets. The final category was included for tweets that used the aforementioned hashtags but mentioned content entirely unrelated to Covid-19. The overall best fitting category was selected if multiple classifications were possible, and consensus discussion was allowed if needed. Once categorized, we calculated the number and percentage of 


\section{Contributors, original research, and FOAM content}

We determined the demographics of Covid-19 FOAM contributors by reviewing 1000 random profiles of the contributors whose tweets were captured in the search strategy, extracting: the number of followers, total tweets, country of residence, and contributor source (institution, nurse, staff physician, resident physician, medical student, respiratory therapist, pharmacist, other healthcare professional, non-healthcare professional, non-clinician researcher, healthcare-related group). The profiles were randomized and selected using the randomize function in Microsoft Excel (Microsoft Corporation, Redmond, WA). We performed a similar analysis on the contributor profiles whose tweets were flagged as blatantly false or misleading.

To evaluate the dissemination of original research via Twitter, we analyzed the journal of publication, country of the corresponding author, article type (epidemiological study, intervention study, diagnostic study, basic science, case series, or other), and the median number of days between online publication (either pre-publication or online) and tweet for each included article. To ensure a focus on new research being conducted on Covid-19 (as opposed to previous coronavirus infections), we excluded research articles published before 2020 .

We also identified tweets that linked to FOAM resources and the source (website), type of resource, number of tweets, including the resource, and the median number of days between FOAM publication online and the tweet.

\section{Data analysis}

We saved the extracted data in Microsoft Excel 2013 (Microsoft Corporation) and analyzed it using R version 3.6.2 (R Project for Statistical Computing, R Core Team, Vienna, Austria). When appropriate, we assessed the distribution of our data using a Shapiro-Wilks test and calculated the mean (+/- standard deviation) for normally distributed data and median (1st and 3rd interquartile range) for data that were not normally distributed. A post-hoc Mann-Whitney U test was performed to compare the days between publication of original research or FOAM resource and tweet. A post-hoc Mann-Kendall trend test with a Bonferroni correction was used to assess for a trend in the percentage of total tweets represented by each content category per week. Inter-rater reliability was assessed using Fleiss' Kappa. Statistical significance was set at a p-value of less than or equal to $0 \cdot 05$.

\section{Results}

The first tweet matching the search criteria was on January 19, 2020, and from then until March 21, 2020, 74,758 original tweets and retweets from 52,917 contributors were created. Of these, 8819 (11.8\%) were original tweets created by 4104 contributors, and 65,490 (88.2\%) were retweets (Table 1). We excluded 278 tweets because they were not written in English or contained broken links. Of the remaining 8541 (11.4\%) original tweets, 5494 were standalone tweets, 1039 were replies, and 2008 were retweets with comments (Figure 1). The original tweets and retweets reached 95,072,663 Twitter users and had a total of 388,701 likes or replies. Contributors to original tweets had a median number of 489 (interquartile range (IQR) 144, 1601) followers and published a median of one (IQR 1,2) original tweet. A Shapiro-Wilks test showed the data was non-parametric $(\mathrm{p}<0.001)$. 


\section{Cureus}

Characteristics

Original tweets and retweets (total)

Retweets without comments

Original tweets (included)

-Standalone tweets

-Replies

-Retweets with comments

Median number of original tweets per original contributor (IQR)

Total reach (number of unique people who saw the tweet)

Total impressions (number of likes and retweets)

Total contributors (tweets and retweets)

Original contributors

Median followers per original contributor (IQR)

Language (tweets and retweets)

English

Unclassified

Spanish

German

French
Number (\%)

$74,758(100 \%)$

$65,940(88 \cdot 2 \%)$

$8541(11 \cdot 4 \%)$

5494

1039

2008

$1(1.2)$

$95,072,663$

388,701

52,917

4104

489 (IQR 144, 1601)

$72,927(97.6 \%)$

$909(1.2 \%)$

$560(0.7 \%)$

$124(0.2 \%)$

$45(0.1 \%)$

TABLE 1: Characteristics of total tweets, retweets, and contributors

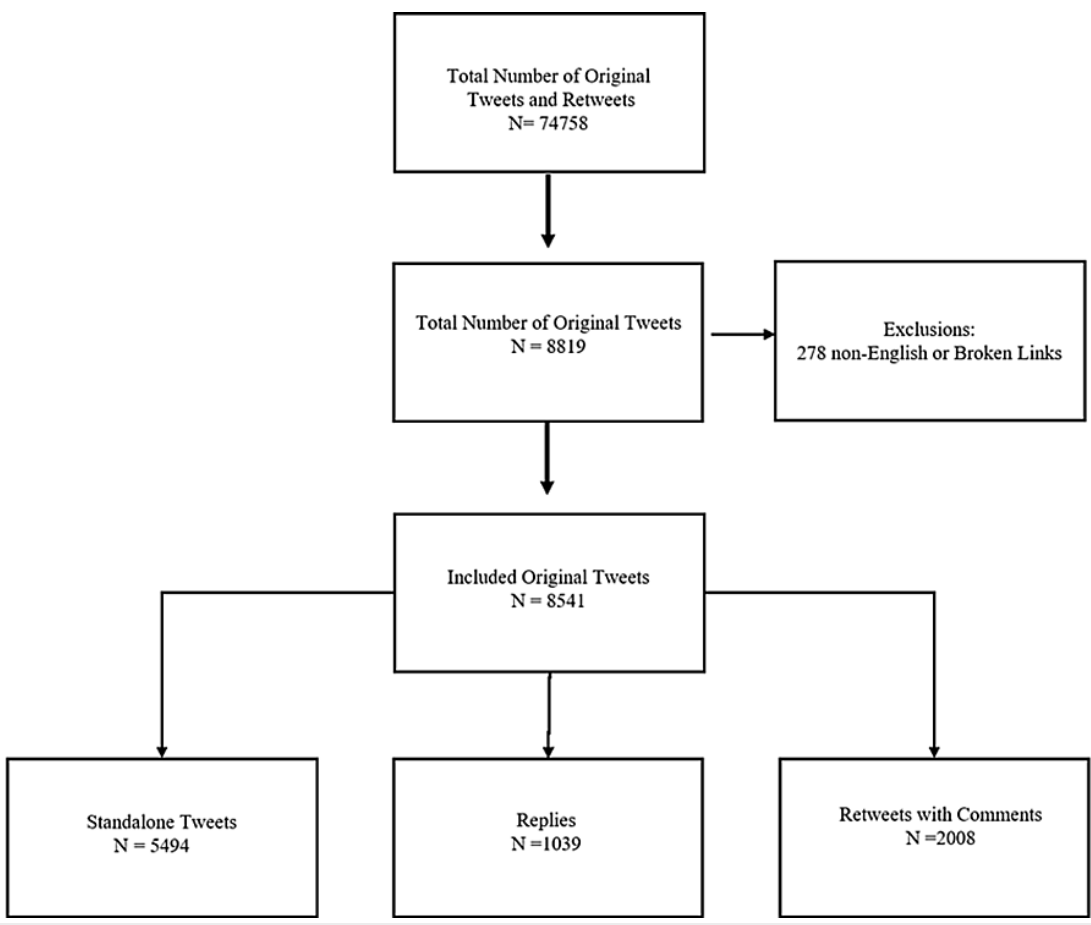

FIGURE 1: Flow diagram from included tweets 


\section{Cureus}

\section{Content analysis}

The number of tweets per day in each content category is displayed in Figure 2. The tweets per week are displayed in Appendix 1. Most Covid-19-related tweets (1557 or 18.2\%) did not fall into one of the predetermined categories. There were 1122 tweets sharing FOAM resources, including blog posts (13.1\%), 275 sharing peer-reviewed original research articles (3.2\%), 304 sharing non-research journal articles (editorials, commentaries, or perspectives) (3.6\%), 410 sharing podcasts or videos (4.8\%), and 245 linking to public health agencies or university websites (2.9\%). There were also 1190 tweets discussing emotional impact (13.9\%), 1111 tweets discussing public policy (13.0\%), 928 tweets asking for advice pertaining to Covid-19 patients (10.9\%), and 873 tweets about learned or personal experience caring for Covid-19 patients (10.2\%). In addition, there were 83 tweets refuting false information (1.0\%), 19 tweets providing blatantly false or misleading information (0.2\%), and 424 non-Covid-19-related tweets (5.0\%). There was no significant change in the percentage of total tweets represented by each content category over time (see supplementary materials). Overall, interrater reliability was fair with a kappa of 0.31 . A selection of representative tweets is provided in Table 2.

$$
1200
$$

1000

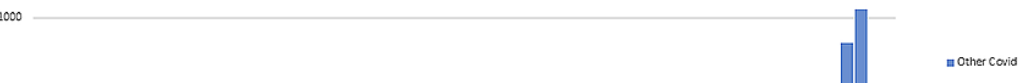

800
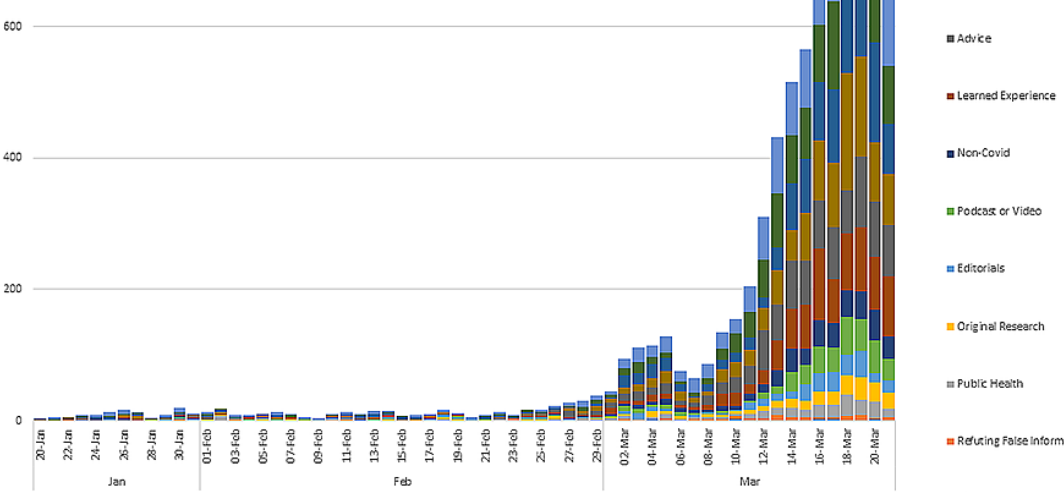

\# Pocteas or Vides

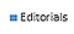

w Orighal Research

FIGURE 2: Total number of original tweets by content category per week 


\section{Cureus}

Content

Category

Link to

FOAM

resources

Asking for

advice

Link to

original

research

Blatantly

false

information

Other Covid-

19 related

discussion

Sharing

personal

experience

Emotional

Impact

Tweet

Free Critical care training modules from @SCCM for non-ICU clinicians to prepare \#medtwitter \#COVID19 \#covid4MDs

$\mathrm{Hi}$, primary care doctor at a major NYC hospital here. Now that's there's community transmission of COVID-19, how do we treat patients with mild respiratory symptoms(cough and fever) who have not had contact with known COVID19 cases? \#medtwitter \#COVID-19 @CDCgov 1/5

Remdesivir and chloroquine effectively inhibit the recently emerged novel coronavirus (2019-nCoV) in vitro https://t.co/WIBwkSNS9n \#coronavirus \#covid19 \#medtwitter \#internalmedicine

REPORT: \#CoronaVirus is a bio-weapon experiment gone wrong, suspect global experts. \#breakingnews \#medtwitter \#covid19 \#wuhan \#china

1) Randomized clinical trials are important. Promising doesn't mean it will work. We have seen other promising medicines fail in trials. 2) We have a chloroquine shortage. MGH has been using hydroxychloroquine instead. \#medtwitter \#covid19

Reminder that how you take OFF PPE is very important so u don't shower yourself w virus. Clutch gown at chest, pulls down over gloves, pull off with gloves, wash hands, pull off mask and goggles straight away from face (NOT UP); wash hands. \#COVID19 \#coviddoc \#medtwitter

As the world is shaken, it turns to us. We can't be shaken. We have an opportunity to be the beacon that guides the world. That's rare and wonderful. In midst of uncertainty/fear, there is no other place l'd rather be. Proud to be in \#healthcare \#COVID19 \#medtwitter \#intensivist

\section{TABLE 2: Sample of original tweets included in the study}

\section{Contributor demographics}

The one-thousand randomly sampled contributors published 2464 tweets, which were cast to a total of 2,618,061 followers (Table 3). Their median number of followers was 455 (IQR 136, 1582). Contributors included 437 staff physicians (43.7\%), 77 resident physicians (7.7\%), 73 non-healthcare professionals $(7.3 \%)$, 68 medical students (6.8\%), and 78 nurses and other healthcare professionals (7.8\%), and 126 (12.6\%) indeterminate. Of the 1000 randomly-sampled contributors, 614 were from North America (61.4\%), 100 were from Europe (10.0\%), 26 were from Australia or New Zealand (2.6\%), 24 were from Asia (2.4\%), six were from South America (0.6\%), five were from the Middle East (0.5\%), four were from Africa (0.4\%), and 221 (22.1\%) indeterminate. 


\section{Cureus}

\begin{tabular}{|c|c|}
\hline Source/Profession & Number (\%) \\
\hline Physician & $437(43.7)$ \\
\hline Not Reported & $126(12.6)$ \\
\hline Institution or Organization & $114(11.4)$ \\
\hline Resident Physician & $77(7.7)$ \\
\hline Non-healthcare professional & $73(7.3)$ \\
\hline Medical student & $68(6.8)$ \\
\hline Other Healthcare Professional & $45(4.5)$ \\
\hline Nurse & $31(3.1)$ \\
\hline Non-clinician researcher & $27(2.7)$ \\
\hline Pharmacist & $2(0.2)$ \\
\hline \multicolumn{2}{|l|}{ Location of Contributor } \\
\hline North America & $614(61.4)$ \\
\hline Not Reported & $221(22.1)$ \\
\hline Europe & $100(10.0)$ \\
\hline Australia or New Zealand & $26(2.6)$ \\
\hline Asia & $24(2.4)$ \\
\hline South America & $6(0.6)$ \\
\hline Middle East & $5(0.5)$ \\
\hline Africa & $4(0.4)$ \\
\hline
\end{tabular}

\section{TABLE 3: Demographics of 1000 randomly selected contributors}

Of the 19 contributors who produced tweets flagged as blatantly false or misleading, two (10.5\%) were staff physicians, three (15.8\%) were organization or institutions, two were nurses (10.5\%), one (5.3\%) was a nonclinician researcher, one (5.3\%) was a non-healthcare professional, and 10 (47.4\%) were undetermined. Their median number of followers was 728 (IQR 189, 1145).

\section{Dissemination of original research}

There were 275 tweets that linked to 157 unique peer-reviewed original research articles. Of these, 23 were published before January 1, 2020, and were excluded from the analysis. The 134 remaining studies included 18 epidemiological studies (13.4\%), four intervention studies (3.0\%), four diagnostic studies (3.0\%), 10 basic science studies (7.5\%), and 98 case series or other (73.1\%). The top five most common countries of corresponding authors were China (57, 42.5\%), the United States of America (29, 21.6\%), Australia (11, $8.2 \%)$, the United Kingdom $(15,11.2 \%)$, and Italy $(8,6.0 \%)$. The top five most common journals of publication were The Lancet (20, 14.9\%), The Journal of the American Medical Association (18, 13.4\%), The Lancet Respiratory Medicine (9, 6.7\%), The New England Journal of Medicine (8, 6.0\%), and The Medical Journal of Australia (8, 6.0\%). The median number of days between publication and tweet was 2 (IQR 1,5$)$. Appendix 2 lists the individual studies.

\section{Dissemination of FOAM resources}

There were 1122 tweets containing links to FOAM resources (websites, blogs, infographics, or attached files). The top 10 FOAM resources included in original tweets were "onepagericu.com" (47, 4.2\%), "emcrit.org" (32, 2.9\%), "esicm.org” (28, 2.5\%), "sccm.org” (23, 2.0\%), "butterflynetwork.com” (17, 1.5\%), "rebelem.com” (13, $1.2 \%)$, "elso.blog” (12, 1.1\%) "insightplus.mja.com.au” (12, 1.1\%), "ultrasoundtraining.com.au" (12, 1.1\%), "intensiveblog.com" (11, 1.0\%), and "propofology.com” (11, 1.0\%). The median number of days from publication of a FOAM resource to dissemination on Twitter was one (IQR 0, 3). Many of the resources that were shared were singular attachments that did not contain a link to a FOAM website, blog, or resource. These included 79 documents (eg, hospital Covid-19 protocols), 83 unsourced infographics, and 27 webinars 
(including participant notes). The median number of days between publication of FOAM resources and the tweet was shorter than for original peer-reviewed research (1 vs. $2, \mathrm{p}<0 \cdot 0001)$.

\section{Discussion}

Covid-19 is not the first pandemic where Twitter has played an important role in sharing information. The H1N1 pandemic and Ebola epidemic were widely discussed on Twitter, with millions of original tweets [1213]. When compared to the H1N1 pandemic in 2009, however, the number of tweets discussing Covid-19 tweets has increased exponentially, surging in early March 2020 (Figure 2) [13]. For context, at that time, Europe had Covid-19 cases across the continent, with several hundred deaths in Italy alone [14]. North America also had its first significant outbreak in Washington, USA [15]. Although the FOAM community has contributors worldwide, most tweets were created by North American contributors, which may account for the temporal association between Twitter use and western outbreaks of Covid-19.

Given the immediacy and reach of social media, the FOAM community may be ideally situated to share medical resources during a pandemic. We found that more than one in four tweets contained a link to a Covid-19 resource. Compared to traditional publication peer review, the publication and editorial processes of FOAM resources vary $[10,16]$. FOAM relies on transparent and open post-publication peer review where other contributors can discuss, critique, and sometimes even contribute to resources [7]. One illustration of effective post-publication peer review during the Covid-19 pandemic has been the Internet Book of Critical Care (IBCC) chapter on Covid-19 [17]. From March 2 to April 16, 2020, the IBCC received over $2 \cdot 1$ million views, with over 180 comments contributing to post-publication peer review [18]. This has led to numerous revisions of the chapter to incorporate new evidence. To consolidate and share the vast amount of information being generated during a pandemic, open post-publication peer review may help balance the timely dissemination of content whilst ensuring its accuracy and quality.

In addition to the speed and reach of FOAM publication, social media may be particularly effective in sharing tacit knowledge [9]. This stems in part from its ability to facilitate discussions and story-telling, which are key components of tacit knowledge translation [9]. During Covid-19, the geographic progression of the disease from Asia, to Europe, to North America allowed for clinicians to share their experiences managing Covid-19 patients. In our study, we found that $9.8 \%$ percent of original tweets shared learned experiences and $10 \cdot 8 \%$ represented individuals asking for crowd-sourced advice. Many of these early tweets pertained to the diagnosis of Covid-19 infection, ultrasound use, airway management, personal protective equipment, and mechanical ventilation. These resources may have helped clinicians and organizations to prepare for Covid-19.

With the unprecedented amount of new Covid-19 research being published, it seems increasingly difficult to find accurate and reliable content online. This has been termed an "infodemic' by the WHO [19]. Although Twitter might contribute to this through the indiscriminate sharing of information, it can also flag important new research and give clinicians a forum to openly critique it. In our study, we found that contributors rapidly tweeted new Covid-19 research, with a median time between publication and tweet of two days. The majority were published in high-impact journals and included important early studies on Covid-19. The immediacy of discussion and rebuttal provided through Twitter also may be valuable, especially when many studies are first being published as pre-prints and have not undergone peer review. For example, when Gautret et al. published their initial pre-print advocating for the use of hydroxychloroquine and azithromycin as a treatment for Covid-19 [20], many FOAM contributors called for more rigorous studies with patient-important outcomes before widespread adoption (Table 2). Their criticisms were substantiated with recent studies showing increased adverse events and a potential association with mortality for hydroxychloroquine [21-22].

Contributing to and participating in the FOAM community is not without risks, and the unwritten rule is caveat emptor (buyer beware). Across social media, the potential for receiving misinformation is real and significant [23]. In this study, 19 contributors contributed blatantly false or misleading information; however, this represented only $0 \cdot 2 \%$ of the total number of tweets in this analysis. Whereas blatantly misleading tweets are relatively easy to identify, a significant concern is when a reader is misled through either misrepresentation of opinion as fact, sensational anecdotes, or providing content without context. Furthermore, subtly misleading or incorrect information may prove much more challenging to interpret and is a serious and ongoing concern when using open-sourced information. A study by Kouzy et al. found that one in four tweets about Covid-19 across Twitter (no FOAM hashtags) contained misinformation [24]. We suspect the rate of subtle misinformation in the FOAM community is higher than the $0 \cdot 2 \%$ found in this study; however, given that the community's collective goal is to share legitimate knowledge, it is likely lower than the broader Twitter community.

FOAM has the potential to decrease the knowledge translation gap during Covid-19; however, resources may be of variable quality [25]. Readers are responsible for critically appraising online content; however, locating quality resources, to begin with, can be a challenge. The Social Media Index (SMI) provides a list of FOAM websites that are both impactful [26] and high quality [27], analogous in some ways to a journal's impact factor. When reading these resources, the Academic Life in Emergency Medicine (ALiEM) AIR tool or revised Medical Education Translational Resources: Impact and Quality (METRIQ) tool has been validated to help 
determine the quality of content [28-29]. Readers may use these tools as an aid when navigating unfamiliar FOAM resources to better appraise the quality of the online resource.

Despite potential pitfalls from engaging with the FOAM community, it is a vibrant community that contributes to the timely dissemination and translation of medical knowledge throughout the world. Few other media, if any, are capable of sharing information, crowdsourcing advice, and providing warnings more rapidly; again, supported by the fact that new articles and FOAM resources discussing Covid-19 were shared within several days of online publication. In situations where efficient communication is essential, such as in global pandemic, natural disasters, or political upheavals, the benefits of such a system may outweigh the possibility of misleading information. It is also important to recognize that papers published in traditional print journals are not immune to misleading information and bias, and may have their own 'Spin' or biased interpretation of results. Furthermore, Twitter allows for tailored advice to individuals through the ability to share personal experiences and ask specific questions, as was seen in our study. With these potential benefits, we feel that Twitter FOAM augments knowledge translation achieved through conventional scientific publications and will continue to do so in the years to come.

\section{Limitations}

Our study has several limitations. Although a broad hashtag search strategy was used, some FOAM hashtags were not included. As well, many Twitter contributors do not add hashtags to all of their Tweets, meaning that some tweets that would have been relevant were missed. For example, it is possible that certain tweets with relevant medical information containing only Covid-19-related hashtags could have been missed. Additionally, although the search was up to date as of March 21, 2020, the necessary time for analysis and manuscript preparation means it does not reflect current Twitter use. As well, inter-rater reliability was only fair, likely related to the fact some tweets fell into several categories. This may account for the reason why a fair proportion of tweets were classified as “other Covid-19." Finally, during the initial months of Covid-19, the largest number of cases were in Asia and Europe [1,30], yet the study was limited to English tweets, potentially selecting for a North American or Anglo-biased perspective.

\section{Conclusions}

In the age of social media, many clinicians use Twitter to share resources and ideas with the goal of improving care for their patients. Twitter is effective in disseminating information; however, it comes with challenges in ensuring content is accurate and relevant. This study provides a detailed characterization of early medical tweets during the Covid-19 pandemic and represents a first step in understanding Twitter use among the FOAM community during the Covid-19 pandemic. Further work is required to improve Twitter as a knowledge translation tool both for Covid-19 and future global crises, such that misinformation and bias is minimized and factual knowledge dissemination maximized. Covid-19 has united clinicians around the world, and perhaps more than ever, effective strategies for sharing new ideas, accurate information, and quality research are needed.

\section{Appendices}

\section{Appendix 1}




\section{Cureus}

\begin{tabular}{|c|c|c|c|c|c|c|c|c|c|c|c|}
\hline & Week 1 & Week 2 & Week 3 & Week 4 & Week 5 & Week 6 & Week 7 & Week 8 & Week 9 & $\begin{array}{l}\text { Total } \\
\text { Tweets }\end{array}$ & $\begin{array}{l}\text { P } \\
\text { value* }\end{array}$ \\
\hline Original Research & $1(1.6 \%)$ & $2(2.3 \%)$ & 5 (8.1\%) & $2(2.6 \%)$ & 7 (9.1\%) & 10 (5.1\%) & $28(4.1 \%)$ & $61(2.6 \%)$ & 159 (3.2\%) & 275 & 0.47 \\
\hline Editorials & $2(3.3 \%)$ & $6(6.9 \%)$ & $6(9.7 \%)$ & $7(9.1 \%)$ & $\begin{array}{l}8 \\
(10.4 \%)\end{array}$ & $12(6.1 \%)$ & $27(4.0 \%)$ & $74(3.2 \%)$ & $162(3.2 \%)$ & 304 & 0.25 \\
\hline FOAM Content & $\begin{array}{l}7 \\
(11.5 \%)\end{array}$ & $\begin{array}{l}14 \\
(16.1 \%)\end{array}$ & $\begin{array}{l}9 \\
(14.5 \%)\end{array}$ & $\begin{array}{l}17 \\
(22.1 \%)\end{array}$ & $\begin{array}{l}10 \\
(13.0 \%)\end{array}$ & 14 (7.1\%) & $\begin{array}{l}104 \\
(15.4 \%)\end{array}$ & $\begin{array}{l}252 \\
(10.9 \%)\end{array}$ & $\begin{array}{l}695 \\
(13.9 \%)\end{array}$ & 1122 & 0.6 \\
\hline Public Health & $1(1.6 \%)$ & $8(9.2 \%)$ & $1(1.6 \%)$ & $3(3.9 \%)$ & $3(3.9 \%)$ & $13(6.6 \%)$ & $26(3.9 \%)$ & $57(2.5 \%)$ & $133(2.7 \%)$ & 245 & 0.83 \\
\hline Podcast or Video & $2(3.3 \%)$ & $3(3.4 \%)$ & $4(6.5 \%)$ & $4(5.2 \%)$ & $5(6.5 \%)$ & $3(1.5 \%)$ & $35(5.2 \%)$ & $88(3.8 \%)$ & $266(5.3 \%)$ & 410 & 0.6 \\
\hline Learned Experience & $4(6.6 \%)$ & $8(9.2 \%)$ & $5(8.1 \%)$ & $4(5.2 \%)$ & $4(5.2 \%)$ & $\begin{array}{l}20 \\
(10.2 \%)\end{array}$ & $50(7.4 \%)$ & $\begin{array}{l}247 \\
(10.7 \%)\end{array}$ & $\begin{array}{l}531 \\
(10.6 \%)\end{array}$ & 873 & 0.21 \\
\hline $\begin{array}{l}\text { Refuting False } \\
\text { Informatino }\end{array}$ & $0(0.0 \%)$ & $4(4.6 \%)$ & $4(6.5 \%)$ & $2(2.6 \%)$ & $2(2.6 \%)$ & $1(0.5 \%)$ & $10(1.5 \%)$ & $34(1.5 \%)$ & $26(0.5 \%)$ & 83 & 0.29 \\
\hline Policy Discussion & $\begin{array}{l}7 \\
(11.5 \%)\end{array}$ & $\begin{array}{l}13 \\
(14.9 \%)\end{array}$ & $5(8.1 \%)$ & $\begin{array}{l}10 \\
(13.0 \%)\end{array}$ & $6(7.8 \%)$ & $\begin{array}{l}26 \\
(13.3 \%)\end{array}$ & 79 (11.7\%) & $\begin{array}{l}275 \\
(11.9 \%)\end{array}$ & $\begin{array}{l}690 \\
(13.8 \%)\end{array}$ & 1111 & 0.6 \\
\hline Emotional Impact & $\begin{array}{l}7 \\
(11.5 \%)\end{array}$ & $\begin{array}{l}9 \\
(10.3 \%)\end{array}$ & $\begin{array}{l}7 \\
(11.3 \%)\end{array}$ & $\begin{array}{l}8 \\
(10.4 \%)\end{array}$ & $\begin{array}{l}9 \\
(11.7 \%)\end{array}$ & $\begin{array}{l}23 \\
(11.7 \%)\end{array}$ & 67 (9.9\%) & $\begin{array}{l}376 \\
(16.2 \%)\end{array}$ & $\begin{array}{l}684 \\
(13.7 \%)\end{array}$ & 1190 & 0.18 \\
\hline Blatantly False & $0(0.0 \%)$ & $0(0.0 \%)$ & $0(0.0 \%)$ & $0(0.0 \%)$ & $2(2.6 \%)$ & $1(0.5 \%)$ & $4(0.6 \%)$ & $4(0.2 \%)$ & $8(0.2 \%)$ & 19 & 0.23 \\
\hline Advice & $6(9.8 \%)$ & $2(2.3 \%)$ & $1(1.6 \%)$ & $2(2.6 \%)$ & $3(3.9 \%)$ & $\begin{array}{l}31 \\
(15.8 \%)\end{array}$ & 74 (11.0\%) & $\begin{array}{l}321 \\
(13.9 \%)\end{array}$ & $488(9.8 \%)$ & 928 & 0.23 \\
\hline Other Covid & $\begin{array}{l}20 \\
(32.8 \%)\end{array}$ & $\begin{array}{l}13 \\
(14.9 \%)\end{array}$ & $\begin{array}{l}14 \\
(22.6 \%)\end{array}$ & $\begin{array}{l}14 \\
(18.2 \%)\end{array}$ & $\begin{array}{l}16 \\
(20.8 \%)\end{array}$ & $\begin{array}{l}32 \\
(16.3 \%)\end{array}$ & $\begin{array}{l}136 \\
(20.1 \%)\end{array}$ & $\begin{array}{l}404 \\
(17.5 \%)\end{array}$ & $\begin{array}{l}908 \\
(18.2 \%)\end{array}$ & 1557 & 0.18 \\
\hline Non-Covid & $4(6.6 \%)$ & $5(5.7 \%)$ & $1(1.6 \%)$ & $4(5.2 \%)$ & $2(2.6 \%)$ & $10(5.1 \%)$ & 35 (5.2\%) & $122(5.3 \%)$ & 241 (4.8\%) & 424 & 0.47 \\
\hline Total Tweets Per Week & 61 & 87 & 62 & 77 & 77 & 196 & 675 & 2315 & 4991 & 8541 & \\
\hline
\end{tabular}

TABLE 4: Number of original tweets by content category per week

\section{Appendix 2}

\begin{tabular}{|c|c|c|c|}
\hline PMID & Study Title & Journal of Publication & $\begin{array}{l}\text { Article } \\
\text { Type }^{*}\end{array}$ \\
\hline 25599463 & $\begin{array}{l}\text { Fluid Management With a Simplified Conservative Protocol for the Acute } \\
\text { Respiratory Distress Syndrome }\end{array}$ & Critical Care Medicine & 1 \\
\hline 25903751 & $\begin{array}{l}\text { A cluster randomised trial of cloth masks compared with medical masks in } \\
\text { healthcare workers }\end{array}$ & BMJ Open & 1 \\
\hline 29071828 & Intactness of Medical Nonsterile Gloves on Use of Alcohol Disinfectants & Ann Lab Med & 1 \\
\hline 30336170 & $\begin{array}{l}\text { Comparison of high-flow nasal cannula versus oxygen face mask for } \\
\text { environmental bacterial contamination in critically ill pneumonia patients: a } \\
\text { randomized controlled crossover trial }\end{array}$ & $\begin{array}{l}\text { Journal of Hospital } \\
\text { Infection }\end{array}$ & 1 \\
\hline 31479137 & $\begin{array}{l}\text { N95 Respirators vs Medical Masks for Preventing Influenza Among Health Care } \\
\text { Personnel }\end{array}$ & JAMA & 1 \\
\hline 32178954 & $\begin{array}{l}\text { Anesthetic Management of Patients with COVID } 19 \text { Infections during Emergency } \\
\text { Procedures }\end{array}$ & $\begin{array}{l}\text { Journal of Cardiothoracic \& } \\
\text { Vascular Anesthesia }\end{array}$ & 1 \\
\hline
\end{tabular}




\section{Cureus}

32187464 A Trial of Lopinavir-Ritonavir in Adults Hospitalized with Severe Covid-19

32205204

Hydroxychloroquine and azithromycin as a treatment of COVID-19: results of an open-label non-randomized clinical trial

32049601

Chest CT for Typical 2019-nCoV Pneumonia: Relationship to Negative RT-PCR Testing

32166346

Findings of lung ultrasonography of novel coronavirus pneumonia during the 20192020 epidemic

16115318 Chloroquine is a potent inhibitor of SARS coronavirus infection and spread

17302372 Indomethacin has a potent antiviral activity against SARS coronavirus.

17522231 Clathrin-Dependent Entry of Severe Acute Respiratory Syndrome Coronavirus into Target Cells Expressing ACE2 with the Cytoplasmic Tail Deleted

20430477 Inactivation of influenza A virus H1N1 by disinfection process.

22436202

Cough aerosol in healthy participants: fundamental knowledge to optimize dropletspread infectious respiratory disease management

25224111 Aerosol Dispersion During Various Respiratory Therapies: A Risk Assessment Model of Nosocomial Infection to Health Care Workers

28193191 Effective inhibition of MERS-CoV infection by resveratrol

29511076

Coronavirus Susceptibility to the Antiviral Remdesivir (GS-5734) Is Mediated by the Viral Polymerase and the Proofreading Exoribonuclease

29678452 Ultraviolet Germicidal Irradiation of Influenza-Contaminated N95 Filtering

Facepiece Respirators

Pro-inflammatory monocyte profile in patients with major depressive disorder and 31753725 suicide behaviour and how ketamine induces anti-inflammatory M2 macrophages by NMDAR and mTOR

32020029

Remdesivir and chloroquine effectively inhibit the recently emerged novel coronavirus (2019-nCoV) in vitro

32075877 Cryo-EM structure of the 2019-nCoV spike in the prefusion conformation

In Vitro Antiviral Activity and Projection of Optimized Dosing Design of

32150618 Hydroxychloroquine for the Treatment of Severe Acute Respiratory Syndrome Coronavirus 2 (SARS-CoV-2)

32182409 Aerosol and surface stability of HCoV-19 (SARS-CoV-2) compared to SARS-CoV-1

32192578 COVID-19: consider cytokine storm syndromes and immunosuppression

32194981 Hydroxychloroquine, a less toxic derivative of chloroquine, is effective in inhibiting SARS-CoV-2 infection in vitro

32199469 Prolonged presence of SARS-CoV-2 viral RNA in faecal samples

32237278

Isolation and rapid sharing of the 2019 novel coronavirus (SARS-CoV-2) from the first patient diagnosed with COVID-19 in Australia

25637115 Face touching: a frequent habit that has implications for hand hygiene.

31585142

Mobile phones as fomites for potential pathogens in hospitals: microbiome analysis reveals hidden contaminants

3160759

Influenza vaccination and respiratory virus interference among Department of Defense personnel during the 2017-2018 influenza season.

32064853

The epidemiological characteristics of an outbreak of 2019

novel coronavirus diseases (COVID-19) in China

32105632 Clinical course and outcomes of critically ill patients with SARS-CoV-2 pneumonia in Wuhan, China: a single-centered, retrospective, observational study

32119825 Feasibility of controlling COVID-19 outbreaks by isolation of cases and contacts

\section{NEJM}

International Journal of

Antimicrobial Agents

Radiology

Intensive Care Medicine $\quad 2$

Virology Journal

Antiviral therapy

Journal of Virology 3

Am J Infect Control $\quad 3$

BMC Pulmonary Medicine 3

Hong Kong Med J

BMC Infectious Diseases 3

American Society for

Microbiology

Am J Infect Control

3

EBio Medicine

Cell Research

Science

3

Clinical Infectious

Diseases

NEJM

3

The Lancet

3

Cell Discovery

Lancet Gastroenterology \& Hepatology

3

Medical Journal of

Australia

Am J Infect Control

Journal of Hospital

Infection

4

Vaccine

CMAPH

Lancet Respiratory

Medicine

Lancet Global Health

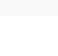

2

(n)

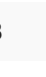

3

3

3

3

3

3

3

3

3




\section{Cureus}

32167524

Risk Factors Associated With Acute Respiratory Distress Syndrome and Death in Patients With Coronavirus Disease 2019 Pneumonia in Wuhan, China

32171076 Clinical course and risk factors for mortality of adult inpatients with COVID-19 in Wuhan, China: a retrospective cohort study

32171390 Real estimates of mortality following COVID-19 infection

32179660 Epidemiology of COVID-19 Among Children in China

32179701

Substantial undocumented infection facilitates the rapid dissemination of novel coronavirus (SARS-CoV2)

32179890 Risk Factors of Healthcare Workers with Corona Virus Disease 2019: A Retrospective Cohort Study in a Designated Hospital of Wuhan in China

15963157 The Impact of Severe Acute Respiratory Syndrome on Medical House Staff

16400088 Concept of Operations for Triage of Mechanical Ventilation in an Epidemic

16885402 A Single Ventilator for Multiple SimulatedPatients to Meet Disaster Surge

19773323 Physical 1s to interrupt or reduce the spread of respiratory viruses: systematic review

23538558 Search for inhibitors of endocytosis

28556555 Examination of Hydroxychloroquine Use and Hemolytic Anemia in G6PDHDeficient Patients

28586113 Lung Consolidation Locations for Optimal Lung Ultrasound Scanning in Diagnosing Pediatric Pneumonia

30368986 Lung-protective Ventilation for Acute Respiratory Distress Syndrome

31766051 Standard and Precordial Leads Obtained With an Apple Watch

31871560 Involving Physicians-in-Training in the Care of Patients During Epidemics

31967327 Emerging coronaviruses: Genome structure, replication, and pathogenesis

31986264 Clinical features of patients infected with 2019 novel coronavirus in Wuhan, China

32004427 First Case of 2019 Novel Coronavirus in the United States

320228362019 Novel Coronavirus - Important Information for Clinicians

32031570

Clinical Characteristics of 138 Hospitalized Patients With 2019 Novel CoronavirusInfected Pneumonia in Wuhan, China

32034323

Mechanisms of action of hydroxychloroquine and chloroquine: implications for rheumatology

32035030 The mental health of medical workers in Wuhan, China dealing with the 2019 nove coronavirus

32052373

Practical recommendations for critical care and anesthesiology teams caring for novel coronavirus (2019-nCoV) patients

32061335

Therapeutic and triage strategies for $\mathbf{2 0 1 9}$ novel coronavirus disease in fever clinics

32066488

Validation of neuromuscular blocking agent use in acute respiratory distress syndrome: a meta-analysis of randomized trials

32066541 Cancer patients in SARS-CoV-2 infection: a nationwide analysis in China

32074258

Preparing for the Most Critically III Patients With COVID-19 The Potential Role of Extracorporeal Membrane Oxygenation

32077115 Clinical characteristics of 140 patients infected with SARS-CoV-2 in Wuhan, China
JAMA Internal Medicine

Lancet

Lancet

4

Pediatrics

4

Science

Clinical Infectious

Diseases

J Gen Intern Med

Academic Emergency

Medicine

5

Academic Emergency

Medicine

5

BMJ

5

Cellular Logistics

5

Arthritis Care Res

5

$\mathrm{J}$ Ultrasound Med

5

Academic Emergency

Medicine

Annals of Internal Medicine 5

Journal of Graduate

Medical Education

5

Journal of Medical Virology 5

Lancet

5

NEJM

5

JAMA

5

JAMA

5

Nature Reviews

5

Lancet

5

Canadian Journal of

Anesthesia

5

Lancet Respiratory

Medicine

5

Critical Care

5

Lancet Oncology

JAMA

5

Allergy 


\section{Cureus}

32085846

Pathological findings of COVID-19 associated with acute respiratory distress syndrome

32085849 The first Vietnamese case of COVID-19 acquired from China

32087116 Asymptomatic cases in a family cluster with SARS-CoV-2 infection

32091533

Characteristics of and Important Lessons From the Coronavirus Disease 2019 (COVID-19) Outbreak in China

32100024 De-isolating Coronavirus Disease 2019 Suspected Cases: A Continuing Challenge

32101510

Correlation of Chest CT and RT-PCR Testing in Coronavirus Disease 2019 (COVID19) in China: A Report of 1014 Cases

32105633 Staff safety during emergency airway management for COVID-19 in Hong Kong

32109013 Clinical Characteristics of Coronavirus Disease 2019 in China

The psychological impact of quarantine and how to reduce it: rapid review of the evidence

32115364

Epidemiological and clinical characteristics of heart transplant recipients during the 2019 coronavirus outbreak in Wuhan china; a descriptive survey report

32125362

Epidemiologic Features and Clinical Course of Patients Infected With SARS-CoV-2 in Singapore.

32125452

Clinical predictors of mortality due to COVID-19 based on an analysis of data of

150 patients from Wuhan, China

32125455

Angiotensin-converting enzyme 2 (ACE2) as a SARS-CoV-2 receptor: molecular mechanisms and potential therapeutic target

Air, Surface Environmental, and Personal Protective Equipment Contamination by

32129805 Severe Acute Respiratory Syndrome Coronavirus 2 (SARS-CoV-2) From a Symptomatic Patient

32133578 Review of the Clinical Characteristics of Coronavirus Disease 2019 (COVID-19)

32134381 Lack of Vertical Transmission of Severe Acute Respiratory Syndrome Coronavirus 2, China

32144127 Coronavirus disease 2019 (covid-19): a guide for UK GPs

32145829 Respiratory support for patients with COVID-19 infection

32146721 An outbreak of COVID-19 caused by a new coronavirus: what we know so far

32150360 Features, Evaluation and Treatment Coronavirus (COVID-19)

32150748

The Incubation Period of Coronavirus Disease 2019 (COVID-19) From Publicly Reported Confirmed Cases: Estimation and Application

32151335

Clinical characteristics and intrauterine vertical transmission potential of COVID-19 infection in nine pregnant women: a retrospective review of medical records

32159735 Care for Critically III Patients With COVID-19

32160316 Co-infection of SARS-CoV-2 and HIV in a patient in Wuhancity, China

32163697 Detection of Covid-19 in Children in Early January 2020 in Wuhan, China

32166318

Protecting Health Care Workers During the COVID-19 Coronavirus Outbreak Lessons From Taiwan's SARS Response

32166346

Findings of lung ultrasonography of novel corona virus pneumonia during the 2019-2020 epidemic

32167525 From Containment to Mitigation of COVID-19 in the US
Lancet Respiratory

Medicine

Lancet Infectious Diseases 5

Lancet Infectious Diseases 5

JAMA

5

Clinical Infectious

Diseases

5

Radiology

5

Lancet Respiratory

Medicine

NEJM

Lancet

Journal of Heart and Lung

Transplantation

JAMA

Intensive Care Medicine

Intensive Care Medicine

JAMA

Jen Intern Med

Emerging Infectious Diseases

BMJ

Lancet

Medical Journal of Australia

Annals of Internal Medicine 5

Lancet

JAMA

5

Journal of Medical Virology 5

NEJM

5

Clinical Infectious Diseases

5

Intensive Care Medicine

5

JAMA 


\section{Cureus}

32167538 Critical Care Utilization for the COVID-19 Outbreak in Lombardy, Italy: Early Experience and Forecast During an Emergency Response

32167853

Can Lung US Help Critical Care Clinicians in the Early Diagnosis of Novel

Coronavirus (COVID-19) Pneumonia?

Are patients with hypertension and diabetes mellitus at increased risk for COVID-

32171062

19 infection?

32172175 Clinical considerations for patients with diabetes in times of COVID-19 epidemic

32173110 A systematic review on the efficacy and safety of chloroquine for the treatment of COVID-19

32176257 COVID-19 and the Risk to Health Care Workers: A Case Report

32180175 Safety and efficacy of different anesthetic regimens for parturients with COVID-19 undergoing Cesarean delivery: a case series of 17 patients

An Analysis of 38 Pregnant Women with COVID-19, Their Newborn Infants, and

32180426 Maternal3 Fetal Transmission of SARS-CoV-2: Maternal Coronavirus Infections and Pregnancy Outcomes

32181795 Coronavirus Disease 2019 (COVID-19) in Italy

32182347 Perinatal Transmission of COVID-19 Associated SARS-CoV-2: Should We Worry?

32187458 SARS-CoV-2 Infection in Children

32191259 Characteristics and Outcomes of 21 Critically III Patients With COVID-19 in Washington State

32192578 COVID-19: consider cytokine storm syndromes and immunosuppression

32199075 Health security capacities in the context of COVID-19 outbreak: an analysis of International Health Regulations annual report data from 182 countries

32199938 Catheterization Laboratory Considerations During the Coronavirus (COVID-19) Pandemic: From ACC's 1al Council and SCAI

32203711 Planning and provision of ECMO services for severe ARDS during the COVID-19 pandemic and other outbreaks of emerging infectious diseases

32204907 The Novel Coronavirus 2019 epidemic and kidneys

32224769 Surviving Sepsis Campaign: Guidelines on the Management of Critically III Adults with Coronavirus Disease 2019 (COVID-19)

32237278 Isolation and rapid sharing of the 2019 novel coronavirus (SARS-CoV-2) from the first patient diagnosed with COVID-19 in Australia

32266965 COVID-19 precautions - easier said than done when patients are homeless

32275288 Neurological Manifestations of Hospitalized Patients with COVID-19 in Wuhan, China: a retrospective case series study
JAMA

Radiology

5

Lancet Respiratory

Medicine

Diabetes \& Metabolic

Syndrome: Clinical

Research \& Reviews

Journal of Critical Care

Annals of Internal Medicine 5

Canadian Journal of Anesthesia

Archives of Pathology \&

Laboratory Medicine

5

JAMA

5

Clinical Infectious

Diseases

NEJM

JAMA

Lancet

Lancet

5

Journal of the American College of Cardiology

Lancet Respiratory Medicine

Kidney International

Intensive Care Medicine

Medical Journal of

Medical Journal of Australia

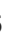

5 . 5 5 . 5 5 5 5 5 5 5

.

${ }^{\star}$ Category: 1 = Intervention 2 = diagnostic 3 = basic science 4 = epidemiological $5=$ case studies, series, or other

TABLE 5: Research articles with Pubmed ID linked in original tweets

\section{Additional Information \\ Disclosures}

Human subjects: All authors have confirmed that this study did not involve human participants or tissue. Animal subjects: All authors have confirmed that this study did not involve animal subjects or tissue. Conflicts of interest: In compliance with the ICMJE uniform disclosure form, all authors declare the 
following: Payment/services info: All authors have declared that no financial support was received from any organization for the submitted work. Financial relationships: All authors have declared that they have no financial relationships at present or within the previous three years with any organizations that might have an interest in the submitted work. Other relationships: All authors have declared that there are no other relationships or activities that could appear to have influenced the submitted work.

\section{Acknowledgements}

All authors contributed to the design of the study, interpretation of data, and manuscript writing. RP, MP, $\mathrm{SB}, \mathrm{KW}$, and NN also performed data analysis. RU and RP performed literature search and figure drafting. RP takes full responsibility for the integrity of the data. All authors have had access to the data and have reviewed the final manuscript.

\section{References}

1. Grasselli G, Zangrillo A, Zanella A, et al.: Baseline characteristics and outcomes of 1591 patients infected with SARS-CoV-2 admitted to ICUs of the Lombardy Region, Italy. JAMA. 2020, 326:1574-1581. 10.1001/jama.2020.5394

2. Zhou F, Yu T, Du R, et al.: Clinical course and risk factors for mortality of adult inpatients with COVID-19 in Wuhan, China: a retrospective cohort study. Lancet. 2020, 395:1054-1062. 10.1016/S01406736(20)30566-3

3. Bedford J, Enria D, Giesecke J, et al.: COVID- 19: towards controlling of a pandemic . Lancet. 2020, 395:1015-1018. 10.1016/S0140-6736(20)30673-5

4. Peyrin-Biroulet L: Will the quality of research remain the same during the COVID-19 pandemic? . Clin Gastroenterol Hepatol. 2020, 18:P2142. 10.1016/j.cgh.2020.03.054

5. Rosenberg H, Syed S, Rezaie S: The Twitter pandemic: the critical role of Twitter in the dissemination of medical information and misinformation during the COVID-19 Pandemic. CJEM. 2020, 22:1-4. 10.1017/cem.2020.361

6. Thoma B, Chan TM, Paterson QS, Milne WK, Sanders JL, Lin M: Emergency medicine and critical care blogs and podcasts: establishing an international consensus on quality. Ann Emerg Med. 2015, 66:396-402. 10.1016/j.annemergmed.2015.03.002

7. Cadogan M, Thoma B, Chan TM, Lin M: Free Open Access Meducation (FOAM): the rise of emergency medicine and critical care blogs and podcasts (2002-2013). Emerg Med J. 2014, 31:76-77. 10.1136/emermed2013-203502

8. Thoma B, Joshi N, Trueger NS, Chan TM, Lin M: Five strategies to effectively use online resources in emergency medicine. Ann Emerg Med. 2014, 64:392-395. 10.1016/j.annemergmed.2014.05.029

9. Potentials of social media for tacit knowledge sharing among clinicians: preliminary findings . (2012). Accessed: Dec 3, 2020: https://aisel.aisnet.org/acis2012/28/.

10. Azim A, Beck-Esmay J, Chan TM: Editorial processes in Free Open Access Medical Educational (FOAM) resources. AEM Educ Train. 2018, 2:204-212. 10.1002/aet2.10097

11. von Elm E, Altman DG, Egger M, et al.: Strengthening the Reporting of Observational Studies in Epidemiology (STROBE) statement: guidelines for reporting observational studies. BMJ. 2007, 335:806-808. 10.7326/0003-4819-147-8-200710160-00010

12. Odlum M, Yoon S: What can we learn about the Ebola outbreak from tweets? . Am J Infect Control. 2015, 43:563-571. 10.1016/j.ajic.2015.02.023

13. Chew C, Eysenbach G: Pandemics in the age of Twitter: content analysis of Tweets during the 2009 H1N1 outbreak. PLoS One. 2010, 5:14118. 10.1371/journal.pone.0014118

14. Coronavirus disease 2019 (COVID-19). Situation report - 48. World Health Organization . (2020). https://www.who.int/docs/default-source/coronaviruse/situation-reports/20200308-sitrep-48-covid-19.pdf? sfvrsn=16f7ccef_4.

15. Arentz M, Yim E, Klaff L, Lokhandwala S, Riedo FX, Chong M, Lee M: Characteristics and outcomes of 21 critically ill patients with COVID-19 in Washington State. JAMA. 2020, 323:1612-1614. 10.1001/jama.2020.4326

16. Sidalak D, Purdy E, Luckett-Gatopoulos S, Murray H, Thoma B, Chan TM: Coached peer review: developing the next generation of authors. Acad Med. 2017, 92:201-204. 10.1097/ACM.0000000000001224

17. Internet book of critical care. (2020). https://emcrit.org/ibcc/covid19/.

18. Farkas, J., IBCC Covid-19 website traffic. Personal correspondance . (2020). Accessed: April 16, 2020: https://emcrit.org/pulmcrit/covid19/.

19. WHO. Novel coronavirus(2019-nCoV). Situation report - 13 . (2020). https://www.who.int/docs/defaultsource/coronaviruse/situation-reports/20200202-sitrep-13-ncov-v3.pdf.

20. Gautret P, Lagier JC, Parola P, et al.: Hydroxychloroquine and azithromycin as a treatment of COVID- 19: results of an open-label non-randomized clinical trial. Int J Antimicrob Agents. 2020, 56:105949. 10.1016/j.ijantimicag.2020.105949

21. Chen Z, Hu J, Zhang Z, et al.: Efficacy of hydroxychloroquine in patients with COVID- 19: results of a randomized clinical trial [PREPRINT]. MedRxIV. 2020, 10.1101/2020.03.22.20040758

22. Magagnoli JN, Siddharth N, Pereira F, Cummings T, Hardin JW, Sutton S, Ambati J: Outcomes of hydroxychloroquine usage in United States veterans hospitalized with Covid-19. Med. 2020, 1:114-127. 10.1016/j.medj.2020.06.001

23. Dijkstra S, Kok G, Ledford JG, Sandalova E, Stevelink R: Possibilities and pitfalls of social media for translational medicine. Front Med . 2018, 5:345. 10.3389/fmed.2018.00345

24. Kouzy R, Abi Jaoude J, Kraitem A, et al.: Coronavirus goes viral: quantifying the COVID-19 misinformation epidemic on Twitter. Cureus. 2020, 12:e7255. 10.7759\%2Fcureus.7255

25. Grock A, Bhalerao A, Chan TM, Thoma B, Wescott AB, Trueger NS: Systematic Online Academic Resource 


\section{Cureus}

(SOAR) review: renal and genitourinary. AEM Educ Train. 2019, 3:375-386. 10.1002/aet2.10351

26. Thoma B, Sanders JL, Lin M, Paterson QS, Steeg J, Chan TM: The social media index: measuring the impact of emergency medicine and critical care websites. West J Emerg Med. 2015, 16:242-249.

10.5811\%2Fwestjem.2015.1.24860

27. Thoma B, Chan TM, Kapur P, et al.: The Social Media Index as an indicator of quality for emergency medicine blogs: a METRIQ study. Ann Emerg Med. 2018, 72:696-702. 10.1016/j.annemergmed.2018.05.003

28. Chan TM, Grock A, Paddock M, Kulasegaram K, Yarris LM, Lin M: Examining reliability and validity of an online score (ALiEM AIR) for rating Free Open Access Medical Education resources. Ann Emerg Med. 2016, 68:729-735. 10.1016/j.annemergmed.2016.02.018

29. Colmers-Gray IN, Krishnan K, Chan TM, et al.: The revised METRIQ score: a quality evaluation tool for online educational resources. AEM Educ Train. 2019, 3:387-392. 10.1002/aet2.10376

30. Guan WJ, Ni ZY, Hu Y, et al.: Clinical characteristics of coronavirus disease 2019 in China . N Engl J Med. 2020, 382:1708-1720. 10.1056/NEJMoa2002032 